\title{
Definition of the attl1 site of class 1 integrons
}

\author{
Sally R. Partridge, ${ }^{1,2}$ Gavin D. Recchia, ${ }^{1,2}$ Carol Scaramuzzi, ${ }^{1,2}$ \\ Christina M. Collis, ${ }^{1}$ H. W. Stokes ${ }^{2}$ and Ruth M. Hall ${ }^{1}$ \\ Author for correspondence: Ruth M. Hall. Tel: +1 6129490 5162. Fax: +1 61294905005. \\ e-mail: ruth.hall@molsci.csiro.au
}

1 CSIRO Molecular Science, Sydney Laboratory, PO Box 184, North Ryde, NSW 1670, Australia

2 School of Biological Sciences, Macquarie University Sydney, NSW 2109, Australia

\begin{abstract}
Integron-encoded integrases recognize two distinct types of recombination site : attl sites, found in integrons, and members of the 59-base element (59-be) family, found in the integron-associated gene cassettes. The class 1 integron integrase, Intl1, catalyses recombination between attl1 and a 59-be, two 59-be, or two attl1 sites, but events involving two attl1 sites are less efficient than the reactions in which a 59-be participates. The full attl1 site is required for high-efficiency recombination with a 59-be site. It is 65 bp in length and includes a simple site, consisting of a pair of inversely oriented Intl1-binding domains, together with two further directly oriented Intl1-binding sites designated strong and weak. However, a smaller region that contains only the simple site is sufficient to support a lower level of recombination with a complete att/1 partner and the features that determine the orientation of att/1 reside within this region. An unusual reaction between the attl1 site and a 59be appears to be responsible for the loss of the central region of a 59-be to create a potential fusion of two adjacent gene cassettes.
\end{abstract}

Keywords: attI, integron, integrase, site-specific recombination, gene cassettes

\section{INTRODUCTION}

Gene cassettes are small mobile elements that generally include only a single gene and a downstream recombination site, known as a 59-base element (59-be), which allows recognition and mobilization of cassettes (Hall et al., 1991; Recchia \& Hall, 1995; Collis \& Hall, 1992a, b; Collis et al., 1993; Hall \& Collis, 1995, 1998). Gene cassettes are normally found associated with integrons. Integrons encode a site-specific recombinase (IntI integrase) and contain a specific site (attI), located adjacent to the intI gene in the integron (Stokes \& Hall, 1989; Ouellette \& Roy, 1987; Martinez \& de la Cruz, 1990), and cassettes are inserted into the attI site. The simplest route that leads to incorporation of gene cassettes into integrons involves IntI-mediated sitespecific recombination between the attI site in the integron and the 59-be in a circularized cassette (Hall et al., 1991), and both integration and excision of cassettes by IntI 1 have been demonstrated experimentally (Collis \& Hall, 1992a, b; Collis et al., 1993).

Four classes of integron, each encoding a distinct IntI integrase, have been identified (Stokes \& Hall, 1989;

Abbreviations: $2^{\circ} \mathrm{rs}$, secondary recombination site; 59-be, 59-base element; 5'-CS, 5' conserved segment; Ap, ampicillin; $\mathrm{Cm}$, chloramphenicol; IntI1, integrase of class 1 integrons; Nx, nalidixic acid; Sm, streptomycin; Su, sulphamethoxazole; Tc, tetracycline; Tp, trimethoprim.
Sundström \& Sköld, 1990; Arakawa et al., 1995; Clark et al., 1997; Mazel et al., 1998). However, only the reactions catalysed by the IntI1 integrase encoded by class 1 integrons have been studied in detail using an in vivo experimental system devised by Martinez $\&$ de la Cruz (1990). As well as the integrative and excisive reactions between attI1 and a 59-be (Martinez \& de la Cruz, 1990; Recchia et al., 1994; Hall et al., 1999), IntI1 can also catalyse both integrative and excisive recombination events between two 59-be (Martinez \& de la Cruz, 1990; Hall et al., 1991; Stokes et al., 1997). Integrative recombination between two attI1 sites has also been documented (Recchia, 1996; Hansson et al., 1997). Recombination between secondary sites ( $2^{\circ} \mathrm{rs}$ ) and 59-be (Francia et al., 1993, 1997; Recchia et al., 1994; Stokes et al., 1997) or attI1 (Hansson et al., 1997) also occurs at a low frequency.

The architecture of the attI1 site differs from that of the 59-be sites (Fig. 1). The 59-be family comprises a large number of sites that have diverse sequences and lengths, but share some common features (Hall et al., 1991; Collis \& Hall, 1992b; Stokes et al., 1997). All include about $25 \mathrm{bp}$ at each end that conform to consensus sequences that are imperfect inverted repeats of one another. The organization of each consensus region is similar to that of a simple site used by other integrases and comprises a pair of inversely oriented integrase-binding domains, 


\title{
(a) attI1/qacE
}

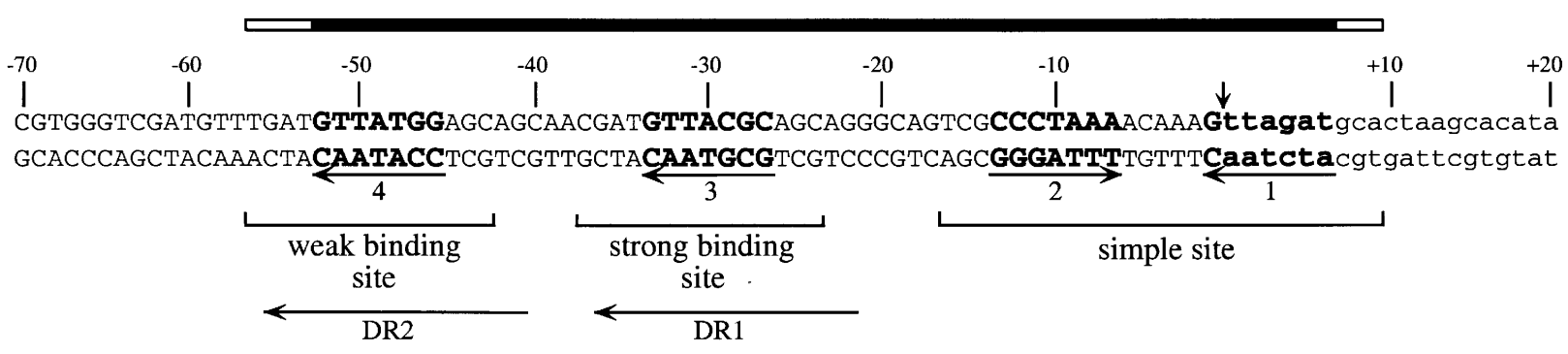

(b) aadB/qacE 59-be

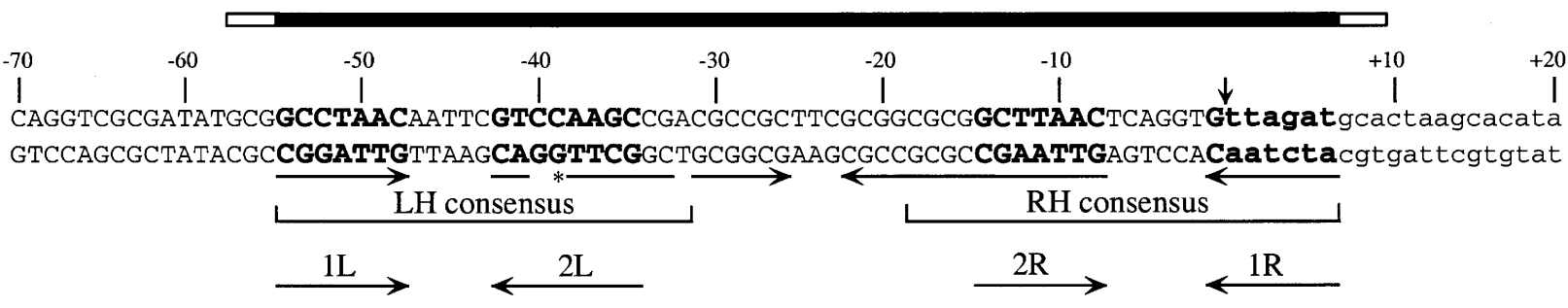

LH simple site

RH simple site

\begin{abstract}
Fig. 1. Sequence and structure of att/1 and a 59-be. The extents of att/1, as determined in this study, and of the 59-be, defined by consensus, are indicated by filled bars. Further bases protected by Intl1 and/or potentially involved in Intl1 recognition (Collis et al., 1998) are shown by open bars. Sequences related to the 7 bp core site are in bold type (1-4 in attI1; IL, 2L, 1R and 2R in the 59-be) and their relative orientations are indicated with arrows. The extents of simple sites are also shown. The recombination crossover points are marked by vertical arrows and the region derived from the qacE cassette is in lower case. (a) The sequence of att/1/qacE shown is from bases 1219 to 1288 and 1880 to 1899 in Cameron et al. (1986). The experimentally determined strong and weak Intl1-binding sites (Collis et al., 1998) and a pair of direct repeats, DR1 and DR2, are indicated. (b) The sequence of the aadB/qaCE 59-be shown is from bases 1810 to 1899 in Cameron et al. (1986). Inverted repeats are underscored with arrows and an asterisk indicates the position of the extra C present in $2 \mathrm{~L}$ as compared with $2 \mathrm{R}$. The extents of the $\mathrm{LH}$ and $\mathrm{RH}$ 59-be consensus sequences as defined by Stokes et al. (1997) are also marked.
\end{abstract}

separated by a spacer of 7 or 8 bp (Stokes et al., 1997; Fig. 1b). In contrast, the attI sites of different integron classes do not share most of these features, nor do they share substantial sequence identity with each other (Recchia et al., 1994; Collis et al., 1998; Hall et al., 1999). For attI1, 2 and 3 only one potential simple-site region can be detected by inspection of the sequences, whereas for attI4 even this simple site is not obvious.

As the 59-be and the attI sites represent two distinct types of recombination site, three formally distinct reactions, attI $1 \times 59$-be, 59 -be $\times 59$-be and attI $1 \times$ attI, are all catalysed by IntI1. The position of the recombination crossover (vertical arrows in Fig. 1) has been localized to between the G and TT in 1R of 59-be (Stokes et al., 1997) and to the equivalent position in site 1 of attI1 (Hansson et al., 1997). IntI1-mediated recombination thus appears to involve only a single strand exchange and the product containing a Holliday junction is presumably resolved by subsequent processes such as replication or the action of a Holliday junction resolvase (Stokes et al., 1997). In an integron that contains cassettes, the 59-be of integrated cassettes will be composite sites in which the sequence following the $G$ residue in $1 \mathrm{R}$ (lower-case letters in Fig. 1) comes from the adjacent cassette or module. Similarly, the attI1 site will also be a composite site in which most of site 1 is derived from $1 \mathrm{R}$ in the 59-be of the first integrated cassette.

The length of the $5^{\prime}$-CS region (in upper case in Fig. 1a) that is required for maximal recombination activity has been delineated experimentally for the attI1 site (Recchia et al., 1994) using an assay that was shown to measure only recombination between attI1 and a 59-be. Maximal activity of the attI1/qacE site was observed with fragments containing at least $64 \mathrm{bp}$ of the $5^{\prime}$-CS, and smaller fragments which include 33 or 31 bp of the $5^{\prime}$-CS had greatly reduced activities (Recchia et al., 1994). However, in a subsequent study where recombination between two attI1 sites was measured (Hansson et al., $1997)$, it was found that no more than 14 bp of the $5^{\prime}$-CS, i.e. only the simple-site region of the attI1 site, was needed for maximal recombination efficiency with a 
complete attI1 site. More recent studies have examined the binding of IntI1 to DNA fragments that include all or part of the attI1 region. As expected, two molecules of IntI1 bind to the simple-site region (Gravel et al., 1998). However, IntI1 binds to two further regions located to the left of the simple site (Collis et al., 1998; Gravel et al., 1998). These binding sites (Fig. 1a), one of which is a strong IntI1-binding site and the other a weak binding site, overlap the direct repeats DR1 and DR2 (Collis et al., 1998; Hall et al., 1999).

In this study the differences in the reported length of the attI1 site have been re-examined. The features of the attI1 site essential for efficient recombination with either a 59-be or a second attI1 site have been assessed using conditions where only one of these reactions can occur. Rare recombinants have been used to more accurately locate the left-hand IntI1-binding domain of the attI1 simple site.

\section{METHODS}

Bacterial strains. Escherichia coli [DH5 $\alpha$ supE44 $\Delta l a c U 169$ ( $\phi 80$ lacZ $\Delta \mathrm{M} 15$ ) hsdR17 recA1 endA1 gyrA96 thi-1 relA1] was used for cloning. Strains UB5201 $\left(\mathrm{F}^{-}\right.$pro met recA56 gyrA) and UB1637 ( $\mathrm{F}^{-}$his lys trp recA56 rpsL) (de la Cruz \& Grinsted, 1982) were used in conduction assays. Bacteria were routinely cultured in LB medium or LB agar supplemented as appropriate with ampicillin (Ap; $100 \mu \mathrm{g} \mathrm{ml}^{-1}$ ), chloramphenicol $\left(\mathrm{Cm} ; 25 \mu \mathrm{g} \mathrm{ml}^{-1}\right)$, nalidixic acid $\left(\mathrm{Nx} ; 25 \mu \mathrm{g} \mathrm{m}^{-1}\right)$, sulphamethoxazole $\left(\mathrm{Su} ; 25 \mu \mathrm{g} \mathrm{ml}^{-1}\right)$, or trimethoprim (Tp; $25 \mu \mathrm{g} \mathrm{ml}^{-1}$ ). Antibiotics were obtained from Sigma.

Plasmids. Plasmids used in this study are listed in Table 1. Fragments containing various lengths of the attI1 recombination site were generated by PCR using pRMH232 as a template and the following primers: RH205, 5'-CGTTACGCCGTGGGTCGACGTTTGAT-3' (attI1 -78 to -53); RH206, 5'-GCTGTGAGCAATTATCAGCTGAGTGC-3' $(q a c E+7$ to +32); RH211, 5'-GGAGCAGCAACGATGTTAC-3' (attI1 - 47 to -29), where bases in bold type indicate changes from the published sequence. These fragments were treated with the Klenow fragment of DNA polymerase I and cloned directly into the unique EcoRV site (GenBank accession no. X06403, position 1680; Rose, 1988) of pACYC184 $\left(\mathrm{Cm}^{\mathrm{R}} \mathrm{Tc}^{\mathrm{R}}\right.$; Chang \& Cohen, 1978; Table 1). The plasmids recovered were sequenced to confirm that they had the required inserts and to determine their orientation. Orientation 1 refers to plasmids in which the right-hand end of each composite site, as shown in Fig. 1, is closest to the origin of replication of pACYC184, while orientation 2 is the opposite. One clone in each orientation was retained. pRMH653 and pRMH654 were identified in this process and presumably arose by spontaneous deletion of part of the RH211/RH206 PCR product. pRMH653 includes only 36 bp

Table 1. Plasmids

\begin{tabular}{|c|c|c|c|c|}
\hline Plasmid & Description & $\begin{array}{l}\text { Position in } \\
\text { pACYC184* }\end{array}$ & $\begin{array}{l}\text { Relevant } \\
\text { phenotype }\end{array}$ & Reference \\
\hline R388 & $\begin{array}{l}33 \mathrm{~kb} \text { IncW plasmid containing In3 (cassettes } \\
d f r B 2 \text {-orfA) }\end{array}$ & NA & $\begin{array}{l}\mathrm{Tp}^{\mathrm{R}} \mathrm{Su}^{\mathrm{R}} \mathrm{Tra}^{+} \\
\quad \mathrm{Int} 1^{+}\end{array}$ & $\begin{array}{l}\text { Avila \& de la Cruz, } \\
\text { (1988) }\end{array}$ \\
\hline pSU2056 & 1176 bp RsaI-BamHI fragment of In2 in pUC9 & NA & $\mathrm{Ap}^{\mathrm{R}} \mathrm{IntI} 1^{+}$ & $\begin{array}{l}\text { Martinez \& de la Cruz } \\
\text { (1990) }\end{array}$ \\
\hline pMAQ76 & 33 bp 5'-CS/198 bp qacE & 1979-EcoRV (1) & $\mathrm{Cm}^{\mathrm{R}}$ & Recchia et al. (1994) \\
\hline pMAQ79 & 31 bp 5'-CS/198 bp qacE & 1986-EcoRV (1) & $\mathrm{Cm}^{\mathrm{R}}$ & Recchia et al. (1994) \\
\hline pRMH232 & 256 bp $5^{\prime}-\mathrm{CS} / 198 \mathrm{bp} q a c E$ & SphI-HindIII (1) & $\mathrm{Cm}^{\mathrm{R}}$ & Collis et al. (1993) \\
\hline pRMH251 & 64 bp 5'-CS/198 bp qacE from pRMH232 & EcoRV (1) & $\mathrm{Cm}^{\mathrm{R}}$ & Recchia et al. (1994) \\
\hline pRMH313 & 64 bp 5'-CS/198 bp qacE from pRMH232 & EcoRV (2) & $\mathrm{Cm}^{\mathrm{R}}$ & This study \\
\hline pRMH560 & Cassette-free derivative of R388 & NA & $\mathrm{Su}^{\mathrm{R}} \mathrm{Tra}^{+} \operatorname{Int} 1^{+}$ & This study \\
\hline pRMH636 & $\begin{array}{l}87 \text { bp PCR fragment (RH219/RH206) of } \\
\text { pMAQ79 in HindII site of pUC19 }\end{array}$ & NA & $A p^{R}$ & Collis et al. (1998) \\
\hline pRMH651 & $\begin{array}{l}110 \text { bp PCR fragment (RH205/RH206) of } \\
\text { pRMH232 }\end{array}$ & $\operatorname{EcoRV}(1)$ & $\mathrm{Cm}^{\mathrm{R}}$ & This study \\
\hline pRMH652 & $\begin{array}{l}79 \text { bp PCR fragment (RH211/RH206) of } \\
\text { pRMH232 }\end{array}$ & $\operatorname{EcoRV}(2)$ & $\mathrm{Cm}^{\mathrm{R}}$ & This study \\
\hline pRMH653 & $\begin{array}{l}68 \text { bp PCR fragment (RH211/RH206) of } \\
\text { pRMH232 }\end{array}$ & $\operatorname{EcoRV}(1)$ & $\mathrm{Cm}^{\mathrm{R}}$ & This study \\
\hline pRMH654 & $\begin{array}{l}45 \text { bp PCR fragment (RH211/RH206) of } \\
\text { pRMH232 }\end{array}$ & $\operatorname{EcoRV}(1)$ & $\mathrm{Cm}^{\mathrm{R}}$ & This study \\
\hline pRMH655 & $\begin{array}{l}109 \text { bp PCR fragment (RH205/RH206) of } \\
\text { pRMH232 }\end{array}$ & $\operatorname{EcoRV}(2)$ & $\mathrm{Cm}^{\mathrm{R}}$ & This study \\
\hline pRMH658 & $\begin{array}{l}79 \text { bp PCR fragment (RH211/RH206) of } \\
\text { pRMH232 }\end{array}$ & $\operatorname{EcoRV}(1)$ & $\mathrm{Cm}^{\mathrm{R}}$ & This study \\
\hline pRMH751 & 87 bp BspHI-HindIII fragment of pRMH636 & BspHI-HindIII (1) & $\mathrm{Cm}^{\mathrm{R}}$ & This study \\
\hline pRMH752 & 81 bp BspHI-SphI fragment of pRMH636 & SphI-BspHI (2) & $\mathrm{Cm}^{\mathrm{R}}$ & This study \\
\hline
\end{tabular}

*Numbers represent positions in pACYC184 according to Rose (1988); numbers in parentheses represent the orientation of the 5 CS/qacE fragment with respect to pACYC184 (see text for details). NA, Not applicable. 
of the $5^{\prime}$-CS and 32 bp of qacE, while pRMH654 has 45 bp of the $5^{\prime}$-CS and none of the $q a c E$ sequence, with base $1(\mathrm{G})$ of the 5 -CS adjacent to the A at position 1682 on the bottom strand of pACYC184 (accession no. X06403; Rose, 1988).

The plasmid pRMH636 contains an attI1/qacE fragment extending from -25 to +32 , derived from $\mathrm{pMAQ} 79$, in the HindII site of pUC19 (Collis et al., 1998). The BspHI site present in the amplification primer RH219 (see Collis et al., 1998) was used in conjunction with either the HindIII site or the SphI site in pUC19 to release the attI1/ qacE fragment from pRMH636. These fragments were cloned into the appropriate sites in pACYC184 to give plasmids pRMH751 and pRMH752, in which the attI1/qacE sequence is present in opposite orientations. These plasmids were sequenced to confirm that they had the required inserts.

The plasmid pRMH560, a derivative of R388 which has lost both the $d f_{r} B 2$ and $\operatorname{orf} A$ cassettes, was generated by IntI1mediated excision of cassettes, as described previously (Collis \& Hall, 1992a).

DNA procedures. Plasmid DNA was isolated using an alkaline lysis method (Birnboim \& Doly, 1979) or a Wizard Miniprep kit (Promega). Restriction enzyme digests were carried out according to the manufacturers' instructions and fragments were separated on $1 \%(\mathrm{w} / \mathrm{v})$ or $0.8 \%(\mathrm{w} / \mathrm{v})$ agarose gels. EcoRI-digested bacteriophage SPP-1 DNA (Bresatec) was used as size markers. BamHI-digested R388 or pRMH560 were also used as markers for mapping cointegrates. DNA fragments for cloning were isolated from agarose gels using a Geneclean II kit (Bio101). Plasmid DNA for sequencing was annealed to primers according to the method of Jones \& Schofield (1990). Double-stranded DNA sequencing was performed using a Sequenase kit, version 2.0 (United States Biochemical) with reaction mixtures containing dITP.

Conduction assays. Recombination efficiency was determined using a conduction (mating-out) assay (Martinez \& de la Cruz, 1988, 1990; Hall et al., 1991; Stokes et al., 1997) to measure conduction of a pACYC184-based test plasmid (containing a cloned attI1 site) from the donor strain UB1637 $\left(\mathrm{RecA}^{-} \mathrm{Sm}^{\mathrm{R}}\right)$ to the recipient strain UB5201 $\left(\mathrm{RecA}^{-} \mathrm{Nx}^{\mathrm{R}}\right)$. Donor cells containing the $\mathrm{Tra}^{+}$plasmid R388 $\left(\mathrm{Tp}^{\mathrm{R}} \mathrm{Su}^{\mathrm{R}}\right)$ or its cassette-free derivative pRMH560 ( $\mathrm{Su}^{\mathrm{R}}$; Table 1) were first transformed with the pACYC184-based test plasmid. pSU2056 $\left(\mathrm{Ap}^{\mathrm{R}}\right.$; Martinez \& de la Cruz, 1990) was then introduced to supply IntI1 integrase in trans. Single colonies of donor strains grown on LB agar were screened for $\mathrm{Ap}^{\mathrm{R}}$ and $\mathrm{Cm}^{\mathrm{R}}$ to confirm that each isolate assayed had a full complement of plasmids after purification.

For matings, $0 \cdot 1 \mathrm{ml}$ portions of stationary-phase cultures of donor and recipient cells were mixed, spread over the surface of an LB agar plate, and incubated overnight at $37^{\circ} \mathrm{C}$. Cells were then harvested and transconjugants selected on agar containing $\mathrm{Tp}$ and $\mathrm{Nx}$ (R388) or $\mathrm{Su}$ and $\mathrm{Nx}$ (pRMH560), while cointegrates were selected on plates containing $\mathrm{Cm}$ and $\mathrm{Nx}$. The conduction frequency was expressed as the fraction of the total transconjugants $\left(\mathrm{Tp}^{\mathrm{R}}\right.$ or $\left.\mathrm{Su}^{\mathrm{R}}\right)$ that contained cointegrates $\left(\mathrm{Cm}^{\mathrm{R}}\right)$. Where possible, three isolates of each donor strain were assayed on at least two occasions and the mean value calculated. For conduction frequencies below $10^{-6}$, where spontaneous mutation of the donor to $\mathrm{Nx}^{\mathrm{R}}$ can contribute significantly to the number of $\mathrm{Cm}^{\mathrm{R}} \mathrm{Nx}^{\mathrm{R}}$ colonies detected, all colonies were screened for $\mathrm{Sm}^{\mathrm{R}}$, which is characteristic of the donor. For a number of cointegrates, the site of insertion of the test plasmid was determined by restriction mapping of the cointegrate DNA with $B a m \mathrm{HI}$ and HindIII, as described previously (Hall et al., 1991; Recchia et al., 1994; Stokes et al., 1997).

\section{RESULTS}

\section{Extent of the attl1 site}

The activity of cloned fragments containing all or part of the attI1 recombination site was tested by measuring cointegrate formation resulting from recombination between the cloned site and a recombination site in the integron In3 found in the conjugative plasmid R388. We have previously shown that when the attI1 fragment is cloned in pACYC184 in one orientation, only products of recombination with the orfA/qacE 59-be, one of three sites in R388, can be recovered (Recchia et al., 1994; Recchia, 1996). This method was used to establish a maximum and minimum for the functional length of attI1 when the partner site is a 59-be (Recchia et al., 1994) and here a wider range of lengths of the 5'-CS were examined in order to establish the role of the strong and weak IntI1-binding sites in attI1 site activity (Table 2). In agreement with the earlier study, attI1/qacE derivatives with either 572 or $64 \mathrm{bp}$ of the $5^{\prime}$-CS each showed a high recombination frequency (approx. $10^{-2}$ ). When all or part of the weak IntI1-binding site located at positions -56 to -43 (Fig. 1a) was removed, leaving only $47 \mathrm{bp}$ (pRMH658) or $36 \mathrm{bp}$ (pRMH653) of the 5'-CS, the recombination frequency was reduced 10 -fold, consistent with a role for this site. Reducing the length of $5^{\prime}$ CS present to $31 \mathrm{bp}$ (pMAQ79) or $25 \mathrm{bp}$ (pRMH751) disrupts the strong IntI1-binding site at -37 to -21 and resulted in a further drop in activity of at least 25 -fold. A further derivative (pMAQ76) with $33 \mathrm{bp}$ of the $5^{\prime}$-CS retains most of the strong binding site and recombination was at an intermediate level. Thus, for the 59be $\times$ attI1 reaction, the strong binding site is critical for efficient attI1 activity and the weak binding site further enhances the efficiency.

The level of recombination activity for the attI1 fragments that contain only the simple site (pMAQ79 and pRMH751) was only slightly higher than that obtained with the vector alone. This background represents recombination between the orfA/qacE 59-be and $2^{\circ}$ rs in the pACYC184-based test plasmid (Recchia et al., 1994). These data confirm that, alone, the simple-site region of attI1 is not an efficient recombination site when the partner site is a 59-be, the activity being equivalent to that of the total of the available $2^{\circ} \mathrm{rs}$ in the test plasmid. However, restriction mapping of 24 cointegrates containing pRMH751 (25 bp of $5^{\prime}$-CS) showed that, despite the low recombination efficiency, the majority of recombinants $(19 / 24)$ had resulted from site-specific events involving the attI1 fragment in pRMH751 and the orfA/qacE 59-be. This indicates that, for recombination with a 59-be, the simple-site region of attI1 is sufficient for IntI1 to recognize this site in preference to any of the available $2^{\circ}$ rs sites.

A further plasmid, pRMH654, contains a fragment that includes bases -45 to -1 of the $5^{\prime}$-CS and hence the strong binding site, but has lost the part of the simplesite region that lies to the right of the recombination crossover (Fig. 1a). This plasmid supports recombination with a 59-be in R388 at a frequency of $7 \cdot 4 \times 10^{-5}$ 
Table 2. Effect of length of $5^{\prime}-\mathrm{CS} / \mathrm{qacE}$ on efficiency of att/1 $\times 59$-be recombination

\begin{tabular}{|lccccc|}
\hline Plasmid* & $\begin{array}{c}\text { Length of } \\
\mathbf{5}^{\prime} \text {-CS/qacE } \\
\end{array}$ & \multicolumn{5}{c|}{ Cointegration frequency } \\
\cline { 3 - 6 } & & No. of assays & Range & Mean & $\%$ \\
\hline pRMH232 & $572 / 198$ & 4 & $6 \cdot 3 \times 10^{-3}-3 \cdot 8 \times 10^{-2}$ & $1 \cdot 6 \times 10^{-2}$ & 106 \\
pRMH651 & $78 / 32$ & 6 & $4 \cdot 4 \times 10^{-3}-1 \cdot 2 \times 10^{-2}$ & $9 \cdot 1 \times 10^{-3}$ & 61 \\
pRMH251 & $64 / 198$ & 6 & $3 \cdot 6 \times 10^{-3}-2 \cdot 8 \times 10^{-2}$ & $1 \cdot 5 \times 10^{-2}$ & 100 \\
pRMH658 & $47 / 32$ & 6 & $6 \cdot 6 \times 10^{-4}-2 \cdot 0 \times 10^{-3}$ & $1 \cdot 3 \times 10^{-3}$ & 9 \\
pRMH653 & $36 / 32$ & 6 & $6 \cdot 5 \times 10^{-4}-2 \cdot 5 \times 10^{-3}$ & $1 \cdot 1 \times 10^{-3}$ & 7 \\
pMAQ76 & $33 / 198$ & 6 & $1 \cdot 4 \times 10^{-4}-6 \cdot 6 \times 10^{-4}$ & $3 \cdot 9 \times 10^{-4}$ & 3 \\
pMAQ79 & $31 / 198$ & 6 & $2 \cdot 9 \times 10^{-5}-7 \cdot 7 \times 10^{-5}$ & $4 \cdot 9 \times 10^{-5}$ & $0 \cdot 3$ \\
pRMH751 & $25 / 32$ & 6 & $2 \cdot 1 \times 10^{-5}-5 \cdot 2 \times 10^{-5}$ & $2 \cdot 9 \times 10^{-5}$ & $0 \cdot 2$ \\
pRMH654 & $45 / 0$ & 7 & $3 \cdot 5 \times 10^{-5}-1 \cdot 5 \times 10^{-4}$ & $7 \cdot 4 \times 10^{-5}$ & $0 \cdot 5$ \\
pACYC184 & - & 6 & $5 \cdot 0 \times 10^{-6}-2 \cdot 5 \times 10^{-5}$ & $1 \cdot 3 \times 10^{-5}$ & $0 \cdot 08$ \\
\hline
\end{tabular}

*Donor strains also contain R388 and pSU2056.

Table 3. Effect of length of $5^{\prime}-\mathrm{CS} / q a c E$ on efficiency of att/1 $\times$ att/1 recombination

\begin{tabular}{|lccccc|}
\hline Plasmid* & $\begin{array}{c}\text { Length of } \\
\mathbf{5}^{\prime} \text {-CS/qacE } \\
(\mathbf{b p})\end{array}$ & No. of assays & Range & Mean & $\%$ \\
\cline { 3 - 6 } & & 5 & $1 \cdot 0 \times 10^{-4}-7 \cdot 9 \times 10^{-4}$ & $4 \cdot 7 \times 10^{-4}$ & 100 \\
& $78 / 31$ & 1 & $1 \cdot 3 \times 10^{-4}$ & $1 \cdot 3 \times 10^{-4}$ & 28 \\
pRMH655 & $64 / 198$ & 6 & $2 \cdot 1 \times 10^{-4}-6 \cdot 1 \times 10^{-4}$ & $3.4 \times 10^{-4}$ & 72 \\
pRMH313 & $47 / 32$ & 5 & $2 \cdot 2 \times 10^{-5}-7 \cdot 5 \times 10^{-5}$ & $5 \cdot 0 \times 10^{-5}$ & 11 \\
pRMH652 & $25 / 32$ & 5 & $2 \cdot 8 \times 10^{-7}-2 \cdot 3 \times 10^{-6}$ & $7 \cdot 7 \times 10^{-7}$ & $0 \cdot 16$ \\
pRMH752 & - & 5 & & & \\
pACYC184 & - & 5 & & & \\
\hline
\end{tabular}

*Donor strains also contain pRMH560 and pSU2056.

(Table 2), which is only slightly higher than that observed with pMAQ79 ( -31 to +198$)$ and pRMH751 $(-25$ to +32$)$. This finding indicates that the presence of the strong binding site is at least as important in determining the efficiency of recombination with a 59-be site as the sequence of the core site (consensus GTTRRRY) surrounding the crossover point, which is replaced by GATCCCG in pRMH654. Mapping of 20 recombinants showed that in half of them recombination had occurred between attI1 in pRMH654 and the orfA/qacE 59-be, indicating that this truncated attI1 site is still recognized in preference to any $2^{\circ}$ rs. Most of the remaining cointegrates appeared to be due to recombination between the orfA/ qacE 59-be in R388 and $2^{\circ}$ rs in $\mathrm{pRMH654.}$

To investigate if the shorter length for attI1 determined by Hansson et al. (1997) is due to the fact that they analysed the attI1 $\times$ attII reaction, the assay used here was modified to measure only recombination between two attI1 sites. Recombinants formed via this reaction are not seen if the fragments are cloned in orientation 1 in pACYC184 but are recovered if the fragment is cloned in the opposite orientation (Recchia, 1996; C. M. Collis, G. D. Recchia \& R. M. Hall, unpublished). Therefore fragments of attI1/qacE with 78, 64, 47 and 25 bp of the
5 '-CS were recloned in the opposite orientation. To ensure that only attI $1 \times$ attI 1 recombination could occur, a derivative of $\mathrm{R} 388$ that has lost the $d f r B 2$ and orfA cassettes (pRMH560), leaving only the attI1/qacE site, was used in place of R388 in the donor strains. The recombination frequency for fragments containing the full attI1 site was at least 20-fold lower than for recombination of the equivalent fragments with a 59-be (Table 3), consistent with a lower efficiency for attI $1 \times$ attI1 events. A small decrease in activity was observed when the fragment length was shortened to include only 25 bp of the $5^{\prime}$-CS (Table 3 ). However, the recombination frequency for this fragment (pRMH752) was still 65-fold higher than that for the vector alone, which in this case represents recombination between the attI1 site in pRMH560 and $2^{\circ}$ rs in the vector. Restriction mapping of 12 cointegrates formed with pRMH752 revealed that, in all of them, recombination had occurred between the two attI1 sites.

\section{The attI1 simple site}

Because the left-hand segment of the attI1 simple site (2 in Fig. 1a) does not contain a region that conforms precisely to the inverse (RYYYAAC) of the 7 bp core site consensus, it is not possible to accurately place this site 


\section{(a)}

$\begin{array}{ll}\text { R388 } & 5^{\prime} \text {-CS } / d f r B 2 \\ \text { B1 } & 5^{\prime} \text {-CS/vector } \\ \text { pACYC184 } & \text { vector } \\ \text { B2 } & \text { vector-dfrB2 } \\ \text { R388 } & 5^{\prime} \text {-CS/dfrB2 }\end{array}$

(b)

$\begin{array}{ll}\text { R388 } & 5^{\prime}-\mathrm{CS} / d f r B 2 \\ \text { B1 } & 5^{\prime} \text {-CS/vector } \\ \text { pACYC184 } & \text { vector } \\ \text { B2 } & \text { vector-dfrB2 } \\ \text { R388 } & 5^{\prime} \text {-CS/dfrB2 } \\ \text { (C) } & \\ \text { R388 } & 5^{\prime} \text {-CS/dfrB2 } \\ \text { B1 } & 5^{\prime}-\text { CS /vector } \\ \text { pACYC184 } & \text { vector } \\ \text { B2 } & \text { vector-dfrB2 } \\ \text { R388 } & 5^{\prime} \text {-CS/dfrB2 }\end{array}$

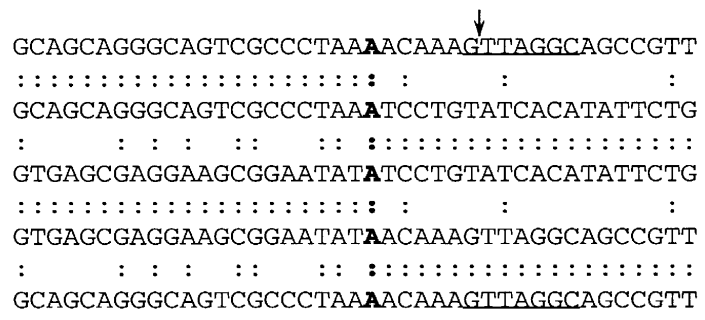

GCAGCAGGGCAGTCGCCCTAAAACAAAGTTAGGCAGCCGTT

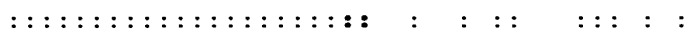
GCAGCAGGGCAGTCGCCCTAAATAAGTGATAATAAGCGGAT : : : : : : : : : : : : : : : : : : : : : : : : : : : : : AACGCCTGGTGCTACGCCTGAATAAGTGATAATAAGCGGAT $:::::::::::::::::::::: \quad: \quad: \quad:: \quad::::$ : $:$ AACGCCTGGTGCTACGCCTGAAACAAAGTTAGGCAGCCGTT

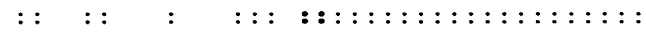
GCAGCAGGGCAGTCGCCCTAAAACAAAGTTAGGCAGCCGTT

(d)

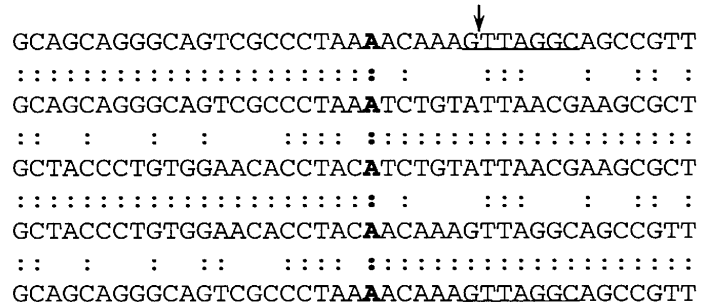
(a)
(b)
(c)
attI1/dfrB2

\begin{abstract}
AGCGGAATATATCCTGTATCACATAT CTACGCCTGAATAAGTGATAATAAGC GAACACCTACATCTGTATTAACGAAG * ** * *

gtcgCCCTAAAACAAAGTTAGGCagc

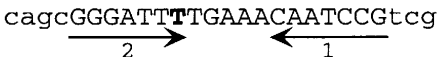

Fig. 2. (a-c) Sequences of junctions in rare cointegrates arising from recombination between the inverse core site (site 2 in Fig. 1a) of att/1/dfrB2 and a $2^{\circ} \mathrm{rs}$ in pACYC184. Each example (a-c) shows the sequence of the $5^{\prime}$-CS/dfrB2 junction in R388 and the relevant part of pACYC184. Amongst the five recombinants recovered, types (b) and (c) were found twice. The att/1/dfrB2 core site (equivalent to 1 in Fig. 1a) is underlined, with the normal crossover point indicated by a vertical arrow. Boundary sequences found in the cointegrates are B1, the boundary between $5^{\prime}$-CS and pACYC184 sequence, and $B 2$, the boundary between pACYC184 sequence and the $d f r B 2$ cassette. Colons indicate identical bases. Bases within the inverse core site and in PACYC184 to which the crossover can be confined are shown in bold type. The positions of these residues in pACYC184 (GenBank accession no. X06403) are: (a) 701 (bottom strand), (b) 3755-4 (bottom strand) and (c) 3487 (top strand). (d) A possible $2^{\circ} \mathrm{rs}$ consensus for recombination with site 2 of attl1. The regions in pACYC184 where recombination occurred are shown aligned with each other and with the att/1/dfrB2 simple site. The bases to which the crossover can be confined are shown in bold type. Bases which are conserved in all three $2^{\circ}$ rs are marked with asterisks. Core sites 1 and 2 of the att/1/dfrB2 simple site are indicated by numbered arrows and bases that are outside the consensus region but predicted to be protected by bound Intl1 are in lower case.

on the basis of sequence alone. Five rare recombinants that arose independently by recombination between the inverse core site (designated 2 in Fig. 1a) of the attI1 simple site and a $2^{\circ}$ rs in the pACYC184 vector were recovered. The sequences of the boundaries in these recombinants (Fig. 2) permit the crossover position to be located to either side of the A at -7 . The inverse core site shown in Fig. 1(a) has been placed in one of the two possible positions on the basis of two criteria. The predicted core site shown differs from the consensus at fewer positions $(2 / 7)$ than the alternative $(3 / 7)$ and the spacer region (defined as the distance between the abnormal and normal crossover locations) is $7 \mathrm{bp}$, which coincides with the spacer length of 7 or 8 bp found in the RH simple site of 59-be (Stokes et al., 1997), rather than $6 \mathrm{bp}$. 
(a)

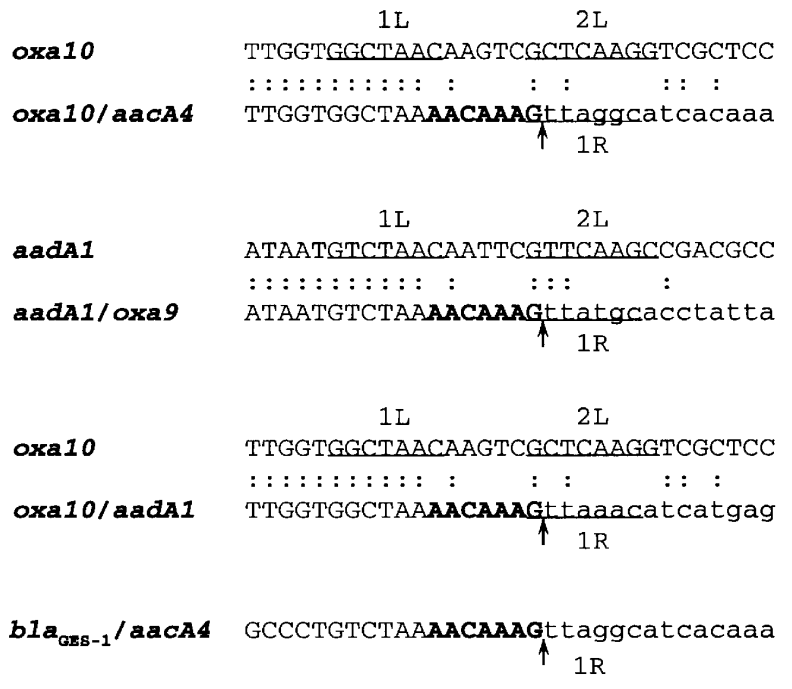

(b)

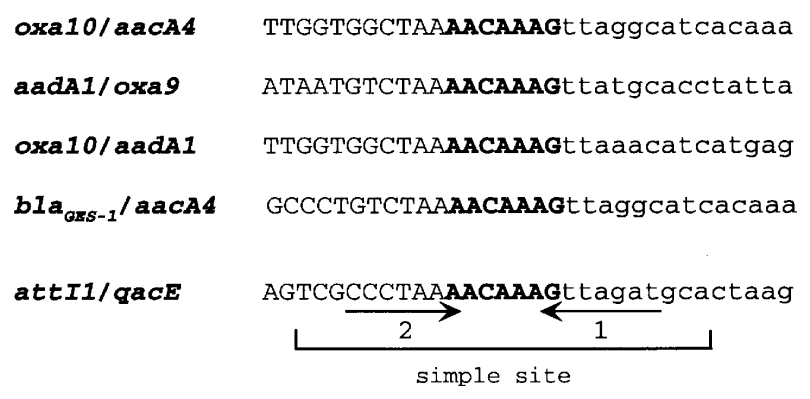

(c)

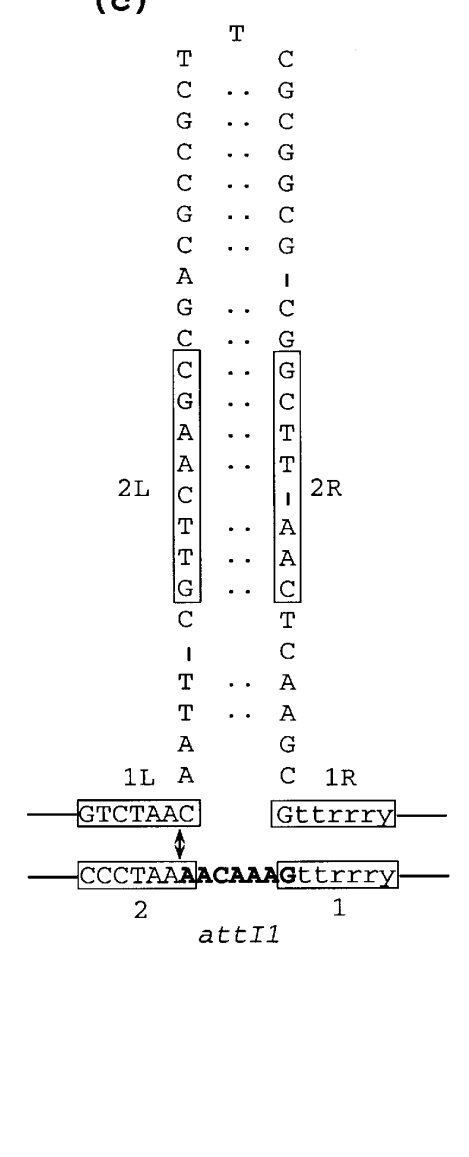

Fig. 3. Replacement of 59-be sequences. (a) Alignment of the sequences of complete and shortened cassettes. The start of the 59-be (1L and $2 \mathrm{~L}$ sites underlined) of the complete cassettes are aligned with sequences at the ends of cassettes that lack a complete 59-be (Tolmasky, 1990; Hall et al., 1993; S. Kupsig \& P. M. Bennett, personal communication; Poirel et al., 2000). For the latter, the first 14 bases of the adjacent cassette are shown in lower case. Colons indicate identity. The position of the normal crossover point, which also marks the end of the integrated cassette, is shown by a vertical arrow. Additional bases not present in the original 59-be are shown in bold type. (b) Comparison of junction sequences with att/1/qacE. The sequences at the boundaries between the shortened cassette and the adjacent cassette are aligned with the simple-site region of att/1/qaCE. The sequence AACAAAG found as the spacer in att/1 and between the two cassettes in the fusion is shown in bold type. The $7 \mathrm{bp}$ core sites in the att/1 simple site are indicated by arrows. (c) A schematic model for the replacement of 59-be central sequences. The aadA1 59-be is shown folded and the four core sites are boxed. $1 \mathrm{~L}$ and $1 \mathrm{R}$ of the 59-be are aligned with the att/1 core sites 1 and 2 (boxed) and the position of the proposed crossover event is marked with a double-headed arrow. Note that though only one strand of the participating sites is shown, Intl1-mediated recombination involves two double-stranded DNA molecules, but only a single strand exchange.

\section{Deletions within 59-be}

A further reaction that appears to involve IntI1-mediated recombination with the attI1 simple site involves the loss of the bulk of the 59-be found at the end of a gene cassette, potentially resulting in fusion of that cassette with the downstream cassette. There are three different cases where a known cassette that includes a complete 59-be has also been found in an alternative form with a shorter site replacing the 59-be (Tolmasky, 1990; Hall et al., 1993; S. Kupsig \& P. M. Bennett, personal com- munication). The relevant regions of the two versions of these cassettes are aligned in Fig. 3(a). This alignment reveals a segment of $7 \mathrm{bp}$ that is not present in the complete cassette. The sequence of this $7 \mathrm{bp}$ segment is the same in all of the shortened cassettes and replaces the bulk of the central region of the 59-be. It is also identical to the sequence of the $7 \mathrm{bp}$ spacer of the attI1 site (Fig. 3b). The normal 59-be is thus replaced by a single simple-site configuration consisting of the original $1 \mathrm{~L}$ site and a 1R site (derived from the adjacent cassette) separated by the $7 \mathrm{bp}$ attI1 spacer. In a further case 
(Poirel et al., 2000), the complete version of the cassette has not yet been found, but only a simple site which includes the attI1 spacer sequence is present. Fig. 3(c) shows a schematic representation of how the substitution of the central region of the 59-be may have occurred. This involves a crossover between two sites that are only rarely the sites of strand exchange, namely $1 \mathrm{~L}$ in the 59-be and 2 in attI1.

\section{DISCUSSION}

The discrepancy between the minimum length of the attI1 site determined by Recchia et al. (1994) and by Hansson et al. (1997) was re-examined and the difference was traced to the nature of the reaction assayed. The biologically important reaction, i.e. attI $1 \times 59$-be, assayed here and by Recchia et al. (1994) is 100-fold more efficient than attI1 $\times$ attI1 recombination and requires the full 65 bp attI 1 site extending from -56 to +9 in Fig. 1 . The less efficient attI $1 \times$ attI1 reaction described here and also by Hansson et al. (1997) requires only the simple-site region of attI1 (Fig. 1a). The requirement for the longer attI1 in the case of recombination with a 59be site is consistent with in vitro binding studies that have shown that the longer region includes two further IntI1-binding domains (Collis et al., 1998; Gravel et al., 1998). It is likely that these two binding sites act as recombination enhancers by retaining the IntI1 recombinase in the vicinity of the simple site. As recombination of attI1 with a 59-be is far more efficient than recombination between two attI1 sites, and is also the biologically important reaction responsible for slotting gene cassettes into an integron, the attI1 site should clearly be defined as the region that includes both the simple site and the two further IntI1-binding domains.

When Hansson et al. (1997) replaced R38841, which is equivalent to pRMH560, with R388 and the attI1 region tested included 36 or more than $200 \mathrm{bp}$ of the $5^{\prime}$-CS, they observed a substantially higher recombination frequency than for the reaction with R $388 \Delta 1$. This is presumably because the more efficient attI $1 \times 59$-be reaction can now occur as well as attI1 $\times$ attI1 recombination. These data are therefore not equivalent to any of the data presented here, where only one of these events is tested at one time. Shorter fragments containing 27, 20 or $14 \mathrm{bp}$ of the $5^{\prime}$-CS tested by Hansson et al. (1997) were at least 30-fold less active. Although these results are in broad agreement with those presented here, the residual recombination level is higher than that described here and presumably represents att $11 \times$ attI 1 recombination, rather than the residual level of att $11 \times 59$-be recombination.

Previous studies have shown that in recombination between two 59-be or between attI1 and a 59-be, the predominant reaction involves the $1 \mathrm{R}$ site in the 59-be and site 1 in attI1 (Martinez \& de la Cruz, 1990; Hall et al., 1991; Recchia et al., 1994; Stokes et al., 1997). Furthermore, only a single strand-exchange event occurs (Stokes et al., 1997) and this feature distinguishes reactions catalysed by the IntI1 integrase from sitespecific recombination events catalysed by other integrases, where two strand-exchange events staggered by $6-8$ bp normally occur (Sadowski, 1993; Hallet $\&$ Sherratt, 1997). The preferential recognition of site 1 in attI1 and $1 \mathrm{R}$ in a 59-be indicates that features of both attI1 and 59-be type sites ensure that the two sites participating in a recombination event are correctly oriented. Those features appear to lie entirely within the simple-site region of attI1, as recombinants arising from a crossover in site 2 of the att 11 simple site, which can be identified by restriction mapping, were not recovered amongst 19 recombinants where the partner site was the orfA/ qacE 59-be or 12 recombinants where the complete attI1 site was the partner.

However, rare recombinants that arose via strand exchange within the $2 \mathrm{R}$ site in one 59 -be and the $1 \mathrm{R}$ site in a second 59-be (Stokes et al., 1997) or the 1L site of a 59-be and the attI1 site 1 (Hall et al., 1999) have been observed previously. In both cases, the sequences of the junctions within the recombinants revealed that only a single strand-exchange event had occurred. This indicates that strand exchange can occur, albeit with very low efficiency, at sites other than $1 \mathrm{R}$ in a 59-be but in both of these cases the crossover is in the normal location in the partner site. Such rare recombinants have been used to locate the $2 \mathrm{R}$ core site of 59-be (Stokes et al., 1997).

A further type of rare recombinant that has arisen via recombination between the left-hand IntI1-binding domain (inverse core site) of the attI1 simple site (2 in Fig. 1a) and a $2^{\circ}$ rs in pACYC184 was recovered here. These recombinants allow a more precise definition of the attI1 simple site than was possible from inspection of the sequence. However, the sequences of the regions in pACYC184 that are involved in the recombination events do not conform to the $\mathrm{Ga} / \mathrm{tTa} / \mathrm{ca} / \mathrm{t}$ (Francia et al., 1993) or Ga/tT (Recchia et al., 1994) consensus for $2^{\circ}$ rs that participate in reactions with 59-be. Although five bases were conserved in each of the three sites identified here (Fig. 2d), further examples are needed to establish if the conservation is significant. The reaction that gave rise to these recombinants is likely to be extremely rare as the frequency for all recombination events involving attI1 and $2^{\circ}$ rs observed here is extremely low (Table 3 ). It is approximately 20 -fold lower than that for a 59-be with $2^{\circ}$ rs (Table 2 ) and similar background frequencies were observed by Hansson et al. (1997). Only two $2^{\circ}$ rs that recombined at the normal location in attI1 (1 in Fig. 1a) have been located by sequencing and both include a region that is closely related to the $7 \mathrm{bp}$ core site consensus (Hansson et al., 1997). Further examples are needed to determine if the consensus for $2^{\circ}$ rs that recombine with attI 1 is the same as that for $2^{\circ}$ rs that recombine with 59-be. The fraction of $2^{\circ} \mathrm{rs}$ recombinants that arise via a crossover at the core site (1) or inverse core site (2) of the attI1 simple site also remains to be determined.

The proposed route that leads to substitution of the attI1 simple-site spacer for the bulk of a 59-be is also 
likely to be extremely uncommon. The schematic model in Fig. 3(c) shows a requirement for an unusual recombination crossover between two core sites (the attI1 site 2 and $1 \mathrm{~L}$ in the 59-be), neither of which is normally the location for strand exchange. Excision of a cassette from a class 1 integron via crossover between $1 \mathrm{~L}$ in the 59-be and site 2 in attI1 would give rise in a single step to a circularized cassette containing the unusual hybrid simple site and leave behind the remainder of the 59-be fused to site 2 of att11. Reintegration of this cassette into a complete attI1 site with the crossover at the normal position would lead to the configuration seen in the known examples. Alternatively, recombination between the $1 \mathrm{~L}$ site of the 59-be in a cassette contained in one integron with the attI 1 site 2 in a second integron would yield similar products in one step. It is likely that the simple site created as a consequence of these events can participate in IntI1-mediated recombination events. However, the efficiency of recombination with either the attI1 site or with a 59-be partner might be expected to be low (equivalent to pRMH751, i.e. -25 to +32 of $5^{\prime}$-CS) and this could provide a strong linkage between the cassette that ends with the simple site and the downstream cassette.

The fact that the architecture of attI1 differs from that of 59-be appears to play a role in ensuring that cassettes are preferentially integrated adjacent to the attI1 site of a class 1 integron. A similar effect is likely to pertain to the attI2, 3 and 4 sites, but the sequences of the known attI sites are not closely related and architecture of these sites has yet to be examined experimentally.

\section{ACKNOWLEDGEMENTS}

S.R.P. and C.S. were supported by a grant from the Australian National Health and Medical Research Council. We thank Mi-Jurng Kim for analysis of one of the recombinants shown in Fig. 2.

\section{REFERENCES}

Arakawa, Y., Murakami, M., Suzuki, K., Ito, H., Wacharotayankun, R., Ohsuka, S., Kato, N. \& Ohta, M. (1995). A novel integron-like element carrying the metallo- $\beta$-lactamase gene

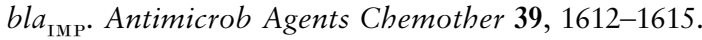

Avila, P. \& de la Cruz, F. (1988). Physical and genetic map of the IncW plasmid R388. Plasmid 20, 155-157.

Birnboim, H. C. \& Doly, J. (1979). A rapid alkaline extraction procedure for screening recombinant plasmid DNA. Nucleic Acids Res 7, 1513-1523.

Cameron, F. H., Groot Obbink, D. J., Ackerman, V. P. \& Hall, R. M. (1986). Nucleotide sequence of the $\operatorname{AAD}\left(2^{\prime \prime}\right)$ aminoglycoside adenylyltransferase determinant $a a d B$. Evolutionary relationship of this region with those surrounding aadA in R538-1 and $d h f r I I$ in R388. Nucleic Acids Res 14, 8625-8635.

Chang, A. C. Y. \& Cohen, S. N. (1978). Construction and characterization of amplifiable multicopy DNA cloning vehicles derived from the p15A cryptic miniplasmid. J Bacteriol 134, 1141-1156.

Clark, C. A., Purins, L., Kaewrakon, P. \& Manning, P. A. (1997). VCR repetitive sequence elements in the Vibrio cholerae chro- mosome constitute a mega-integron. Mol Microbiol 26, 1137-1143.

Collis, C. M. \& Hall, R. M. (1992a). Site-specific deletion and rearrangement of integron insert genes catalysed by the integron DNA integrase. J Bacteriol 174, 1574-1585.

Collis, C. M. \& Hall, R. M. (1992b). Gene cassettes from the insert region of integrons are excised as covalently closed circles. Mol Microbiol 6, 2875-2885.

Collis, C. M., Grammaticopoulos, G., Briton, J., Stokes, H. W. \& Hall, R. M. (1993). Site-specific insertion of gene cassettes into integrons. Mol Microbiol 9, 41-52.

Collis, C. M., Kim, M.-J., Stokes, H. W. \& Hall, R. M. (1998). Binding of the purified integron DNA integrase IntI1 to integronand cassette-associated recombination sites. Mol Microbiol 29, 477-490.

de la Cruz, F. \& Grinsted, J. (1982). Genetic and molecular characterisation of $\mathrm{Tn} 21$, a multiple resistance transposon from R100-1. J Bacteriol 151, 222-228.

Francia, M. V., de la Cruz, F. \& García Lobo, M. (1993). Secondary sites for integration mediated by the Tn21 integrase. Mol Microbiol 10, 823-828.

Francia, M. V., Avila, P., de la Cruz, F. \& García Lobo, M. (1997). A hot spot in plasmid $\mathrm{F}$ for site-specific recombination mediated by Tn21 integron integrase. J Bacteriol 179, 4419-4425.

Gravel, A., Fournier, B. \& Roy, P. H. (1998). DNA complexes obtained with the integron integrase IntI1 at the attI1 site. Nucleic Acids Res 26, 4347-4355.

Hall, R. M. \& Collis, C. M. (1995). Mobile gene cassettes and integrons: capture and spread of genes by site-specific recombination. Mol Microbiol 15, 593-600.

Hall, R. M. \& Collis, C. M. (1998). Antibiotic resistance in gramnegative bacteria: the role of gene cassettes and integrons. Drug Resist Updates 1, 109-119.

Hall, R. M., Brookes, D. E. \& Stokes, H. W. (1991). Site-specific insertion of genes into integrons: role of the 59-base element and determination of the recombination cross-over point. Mol Microbiol 5, 1941-1959.

Hall, L. M. C., Livermore, D. M., Gur, D., Avoka, M. \& Akalin, H. E. (1993). OXA-11, an extended-spectrum variant of OXA-10 (PSE-2) $\beta$-lactamase from Pseudomonas aeruginosa. Antimicrob Agents Chemother 37, 1637-1644.

Hall, R. M., Collis, C. M., Kim, M.-J., Partridge, S. R., Recchia, G. D. \& Stokes, H. W. (1999). Mobile gene cassettes in evolution. Ann N Y Acad Sci 870, 68-80.

Hallet, B. \& Sherratt, D. J. (1997). Transposition and site-specific recombination: adapting DNA cut-and-paste mechanisms to a variety of genetic rearrangements. FEMS Microbiol Rev 21, 157-178.

Hansson, K., Sköld, O. \& Sundström, L. (1997). Non-palindromic attI sites of integrons are capable of site-specific recombination with one another and with secondary targets. Mol Microbiol 26, 441-453.

Jones, D. S. C. \& Schofield, J. P. (1990). A rapid method for isolating high quality plasmid DNA suitable for DNA sequencing. Nucleic Acids Res 18, 7463-7464.

Martinez, E. \& de la Cruz, F. (1988). Transposon Tn21 encodes a RecA-independent site-specific integration system. Mol Gen Genet 211, 320-325.

Martinez, E. \& de la Cruz, F. (1990). Genetic elements involved in Tn21 site-specific integration, a novel mechanism for the dissemination of antibiotic resistance genes. EMBO J 9, 1275-1281. 
Mazel, D., Dychinco, B., Webb, V. \& Davies, J. (1998). A distinctive class of integron in the Vibrio cholerae genome. Science 280, 605-608.

Ouellette, M. \& Roy, P. H. (1987). Homology of ORFs from Tn2603 and from R46 to site-specific recombinases. Nucleic Acids Res 15, 10055.

Poirel, L., Le Thomas, I., Naas, T., Karim, A. \& Nordmann, P. (2000). Biochemical sequence analyses of GES-1, a novel class A extended spectrum $\beta$-lactamase, and the class 1 integron In52 from Klebsiella pneumoniae. Antimicrob Agents Chemother 44, 622-632.

Recchia, G. D. (1996). Mobile gene cassettes and integrons: evolutionary and recombinational studies. $\mathrm{PhD}$ thesis, Macquarie University Sydney, Australia.

Recchia, G. D. \& Hall, R. M. (1995). Gene cassettes : a new class of mobile element. Microbiology 141, 3015-3027.

Recchia, G. D., Stokes, H. W. \& Hall, R. M. (1994). Characterisation of specific and secondary recombination sites recognised by the integron DNA integrase. Nucleic Acids Res 22, 2071-2078.
Rose, R. E. (1988). The nucleotide sequence of pACYC184. Nucleic Acids Res 16, 355.

Sadowski, P. (1993). Site-specific genetic recombination: hops, flips, and flops. FASEB J 7, 760-767.

Stokes, H. W. \& Hall, R. M. (1989). A novel family of potentially mobile DNA elements encoding site-specific gene-integration functions: integrons. Mol Microbiol 3, 1669-1683.

Stokes, H. W., O'Gorman, D. B., Recchia, G. D., Parsekhian, M. \& Hall, R. M. (1997). Structure and function of 59-base element recombination sites associated with mobile gene cassettes. Mol Microbiol 26, 731-745.

Sundström, L. \& Sköld, O. (1990). The $d h f r I$ trimethoprim resistance gene of $\mathrm{Tn} 7$ can be found at specific sites in other genetic surroundings. Antimicrob Agents Chemother 34, 642-650.

Tolmasky, M. E. (1990). Sequencing and expression of addA, bla, and $\operatorname{tnpR}$ from the multiresistance transposon Tn1331. Plasmid 24, 218-226.

Received 31 May 2000; revised 6 August 2000; accepted 14 August 2000. 